\title{
A Cross-Cultural Comparison of the Accuracy of Personality Judgements Made Through Social Media
}

\author{
Mark Turner ${ }^{1}$ and Eeven Chin ${ }^{1}$ \\ ${ }^{1}$ Department of Psychology, University of Portsmouth, Portsmouth, United Kingdom \\ Mark.Turner@port.ac.uk \\ Eeven.Chin@myport.ac.uk
}

\begin{abstract}
The growth of social media has seen a change in the way people meet and communicate. Previous studies have provided mixed evidence for the accuracy of judgments based on social media profiles alone, and relatively little is known about cross-cultural interpretations of online profiles. In the present study, the accuracy of first impressions formed from social media profile pictures was examined for people from similar or different cultural backgrounds. Results showed strong consensus between raters regarding the consistency with which attributes were rated, but poor agreement between raters and profile owners' own ratings of their personality, regardless of cultural background. Some relationships were found between raters' own personality and the ratings they assigned to others suggesting that one's own personality has an impact when making judgments of others. This will become increasingly important as social media expands the possibility of cross-cultural interaction globally.
\end{abstract}

Keywords: First Impressions · Personality · Social Media · Cross-cultural Judgements

\section{Introduction}

It has been suggested a glance as quick as three seconds is all it takes for someone to evaluate another person on first meeting [1]. First impressions are usually formed on factors such as appearance, body language, clothing, and mannerisms. This tendency for humans to make automatic judgments is common across cultures although the circumstances under which such first impressions are formed may be changing. Recently, online social networks have gained popularity and are now a core means of communication in modern society [2]. Social Network Site (SNS) users may spend a large amount of time creating profiles in order to convey aspects of their personality, status or image towards others. It has also become increasingly common to meet people first online rather than face-to-face. Knowledge of how impressions are formed online is, therefore, important to understand how such media can influence the judgments we make of others. The current research asks whether the first impressions formed of others based on their online image are accurate, and how are these impressions influenced by the cultural background of the observer and the profile owner being judged? 
According to Zhao and Jiang [3], self-presentation is strongly influenced by culture. Assumed collectivist cultures are thought primarily to view themselves as part of a whole (i.e. family, tribe, a nation) while more individualist western societies prioritize their own needs rather than group goals [4]. Some early cross-cultural research pointed to differences in the primary motivations for using the Internet. For example, in Hong Kong the internet was perceived as a medium for social interaction, while in America, using the Internet to seek and gain information was more commonly reported [5]. Kim, Sohn and Choi [6] found that SNS are used by Korean students more to gain social support from existing relationships rather than meeting new people, whereas American students used SNS more as a means of entertainment. Given that different cultures seem to have different core motivations for using SNS, then it is also relevant to consider how self-presentation online may also differ in different cultures.

Cultural differences have an impact on how people choose to present themselves to others because the concept of "self" is known to differ by culture [3]. Some studies have shown that Americans and Japanese perceive facial expressions of emotions, in particular smiles, differently [7]. A smile is a rather complex expression of emotion as it can convey genuine positive feelings as well as negative feelings. Moreover, the judgement of smiles differs across different cultures due to differences in cultural display rules [7]. For instance in Japan, people may smile more frequently for social appropriateness rather than true feelings of joy, which may lead the Japanese to perceive fewer emotions in smiles compared to other cultures. Moreover, in the US smiling faces may be associated with positive traits such as friendliness and sincerity whereas in Japan non-smiling faces may be associated with these more positive traits.

When meeting new people, Uleman [8] has proposed that we tend to evaluate their personalities based on our own experience. In other words, we need little new information to form first impression of others. It follows that first impressions formed online may therefore be influenced by past experience of online profiles. Carney, Calvin, and Hall [9] argue that such initial impressions may be based on impoverished information, nevertheless accurate personality judgments can be formed quickly when judging facial images from similar cultural backgrounds. In one study, Naumann, Vazire, Rentfrow and Gosling [10] compared interpretations of participants' in a standard, constrained pose (looking directly at the camera) with participants who posed spontaneously as they wished, finding that judgements of personality were more accurate for spontaneous poses. Whilst some studies have suggested that accurate first impressions may be formed from condensed profile information found online [11], it has also been noted that in some cultures, different display rules for online self-presentation may also exist. For example, Malaysian students adapt information such social connections to construct a desired online identity [12]. Male users were also found to be more comfortable than female users in using their own pictures as their profile pictures. Profile pictures are often carefully selected by profile owners and are one of the first aspects examined by observers of profiles, which has led some others to suggest that a different and evolving set of implied norms exists with respect to online self-presentation [13].

The Internet is now a global technology that has made it easier and more common for people from different countries and cultural backgrounds to interact. Cross-cultural judgments based on online profile are therefore, also becoming increasingly common. Whilst a standard format, that of a profile picture with limited written detail is common 
to most SNS platforms, what is less known is how such public displays may be interpreted by people from different cultural backgrounds.

The focus of this present study is to explore how accurate impressions are formed online from personal images with particular reference to cultural differences. Whilst several studies have explored how first impressions are formed, little is known about how exposure or isolation to people from a different cultural background may impact on judgments of online profiles.

In this study, personality ratings judged from Western (UK) SNS profile owners' pictures are examined and compared to observers' ratings from three cultural groups. It was hypothesized that judges who were drawn from the same cultural background as the profile owners would form more accurate first impressions than judges from different cultural backgrounds.

\section{Method}

\subsection{Design and Participants}

The accuracy of first impressions was explored within three groups: UK based Western university students $(n=40)$, UK based Asian university students $(n=29)$, and overseas based Asian students $(n=27)$ attending university in Malaysia who had never visited the UK. The ages of participants ranged from 18 to 30 years old (Mean age, 20.8; SD=2.1).

All participants were briefly presented with 52 social media profile pictures and were asked to evaluate 10 personality attributes of the profile owner after seeing each picture. Consistent with display durations used in other thin-slice judgment studies [9], pictures were serially presented on separate slides for a set time of 10 seconds. Pictures were shown abstracted from any other social media information about the profile owner.

The 52 female social media profile owners were of similar age to the participant group (Mean age, 19.4; SD, 1.6) and completed a self-evaluation of the same 10 personality attributes. All were Western (UK based) female students, who were not known to the participants in the study, and had given prior consent to their Facebook profile picture being viewed by others [14]. All participants rated the personality characteristics of each profile owner using an adapted version of Bond's dimensions used in perceiving peers scale [15]. This involved the rating of 10 bipolar objectives (e.g., Nervous - Calm) on a 7-point scale where a higher score was associated with the more positive attribute. Personality judgments were compared by using each profile owners' selfevaluation and observer evaluations to create an accuracy score.

\subsection{Procedure}

UK-Based Western Group. Trials were conducted under laboratory conditions. The pictures were serially presented on separate slides for a set time of 10 seconds with a blank slide being shown between pictures during which personality judgments were made. Where pictures contained more than one person, the position of the target individual was stated below the picture. The presentation duration of each picture was controlled by a computer with a researcher present to ensure the task was completed correctly. No discussion of the photographic content of pictures was permitted between the 
researcher and observers. Observers were allowed as much time as they required to complete the personality evaluation for each picture, before moving on to the next picture. Trials took approximately 40 to 50 minutes for participants to complete all 520 separate evaluations (52 targets $\mathrm{x} 10$ ratings).

UK-Based Asian and Overseas Group. The procedures followed for these two groups were essentially the same as the UK based Western group. However, participants from these groups were given a hardcopy of rating scales, rather entering these directly into a separate computer, whilst viewing the 52 profile images. Participants took approximately 30 minutes to complete all ratings in these conditions.

\section{$3 \quad$ Results}

\subsection{Self-other agreement between each groups of raters.}

To examine whether observers from the three cultural groups shared the same level of agreement when judging the personality of the target females, interclass correlations (ICC) were conducted to calculate observer agreement (consensus) for each personality attribute. Cronbach's alpha values for each attribute scored by observers from each group were calculated (Table 1).

The overall findings suggest the level of consensus for averaged raters ICC $(2, \mathrm{k})$ to be best in the UK rater group (alpha range .69 to .94 ) and poorer for the Malaysian raters (alpha range .31 to .92) and UK based Asian group (alpha range 30 to .91). Interestingly, observer agreement for nervousness was the weakest in the UK group, but observer agreement was the lowest for the Malaysian and UK Asian group when judging sensitivity.

Whilst raters in each cultural group generally showed good consensus, accuracy as indicated by self-other agreement based on Pearson correlations of profile owner's ratings of their own personality with averaged ratings of all raters within each cultural group were generally low. In the UK group, only one significant relationship was found out of the 10 attributes; ratings of sensitivity ( $r=.39$ ) was positively correlated between the owners' and raters' ratings, suggesting that raters managed to judge sensitivity with a degree of accuracy. However, no significant relationships were found for the remaining nine attributes, implying that raters' judgments did not agree with the profile owners' evaluations of themselves.

A similar pattern was seen in the Malaysian and UK Asian group where only one significant self-other correlation was found. Both in the Malaysian group $(r=.39)$ and UK Asian group ( $\mathrm{r}=.28$ ), agreement between own ratings and other rater were found with respect to friendliness. This suggests that observers from different cultural backgrounds were better at judging friendliness in Westerners than other Western raters. The findings appear inconsistent with the hypotheses that raters from a similar cultural background would be better at judging the personalities of profile owners, from their profile pictures. 
Table 1. Intraclass correlations for averaged (ICC $2, \mathrm{k}$ ) measures of observer agreement (consensus) and Pearson (r) correlation coefficients between self-ratings and averaged observer ratings for 10 personality attributes $(* \mathrm{p}<.05 * * \mathrm{p}<.01)$.

\begin{tabular}{|c|c|c|c|c|c|c|}
\hline \multirow[t]{2}{*}{ Attribute } & \multicolumn{3}{|c|}{ Consensus (Cronbach's $\alpha$ ) } & \multicolumn{3}{|c|}{ Self-Other Agreement ( $\mathrm{r})$} \\
\hline & $\begin{array}{c}\text { UK } \\
\text { Western }\end{array}$ & $\begin{array}{c}\text { Malay- } \\
\text { sian }\end{array}$ & $\begin{array}{c}\text { UK } \\
\text { Asian }\end{array}$ & $\begin{array}{c}\text { UK } \\
\text { Western }\end{array}$ & $\begin{array}{l}\text { Malay- } \\
\text { sian }\end{array}$ & $\begin{array}{c}\text { UK } \\
\text { Asian }\end{array}$ \\
\hline Nervous-Calm & $.69 * *$ & $.74 * *$ & $.76 * *$ & .21 & .07 & .19 \\
\hline Insecure-Confident & $.92 * *$ & $.83 * *$ & $.80 * *$ & -.16 & -.17 & -.19 \\
\hline Shy-Outgoing & $.94 * *$ & $.85^{* *}$ & $.87 * *$ & .11 & .08 & .08 \\
\hline Unattractive-Attractive & $.94 * *$ & $.92 * *$ & $.89 * *$ & -.06 & -.04 & .01 \\
\hline Unfriendly-Friendly & $.93 * *$ & $.89 * *$ & $.91 * *$ & .22 & $.39 * *$ & $.28 *$ \\
\hline Insensitive-Sensitive & $.90 * *$ & $.31 * *$ & $.30 * *$ & $.39 * *$ & -.03 & .03 \\
\hline Careless-Perfectionist & $.90 * *$ & $.81 * *$ & $.82 * *$ & .06 & .02 & .05 \\
\hline Quiet-Loud & $.94 * *$ & $.90 * *$ & $.89 * *$ & .21 & .02 & .23 \\
\hline Unreliable-Reliable & $.93 * *$ & $.86^{* *}$ & $.85 * *$ & -.13 & .09 & -.07 \\
\hline Unintelligent-Intelligent & $.92 * *$ & $.79 * *$ & $.83 * *$ & -.19 & -.19 & -.21 \\
\hline
\end{tabular}

\subsection{Judgment differences between UK, UK Asian and Malaysian Groups.}

The scores on the 10 personality attributes drawn from each cultural group were analyzed using a one-way MANOVA to determine the differences between three different cultures on how they generally perceived others. The multivariate effect produced from this analysis was significant $(\mathrm{F}(2,93)=2.54, \mathrm{p}<.01$, Wilks' $\lambda=.23)$. Consideration of the univariate statistics, following MANOVA produced six significant univariate effects out of the ten personality attributes. Of the six effects, ratings of confidence $(p<.01)$, calmness $(p=.02)$, outgoingness and quietness $(p=.03)$, friendliness and being a perfectionist $(p=.05)$ were found to differ as a function of cultural background.

Figure 1 shows mean scores of each attribute in each of the three cultural groups. Results suggested a general trend whereby Malaysian based students tended to rate profile owners more conservatively than UK or UK based Asian students. UK based Asian students perceived profile owners as generally being calmer, more confident, more of a perfectionist, and quieter than rates in the other two cultural groups. UK raters considered profile owners to be friendlier than students in the other two groups. 


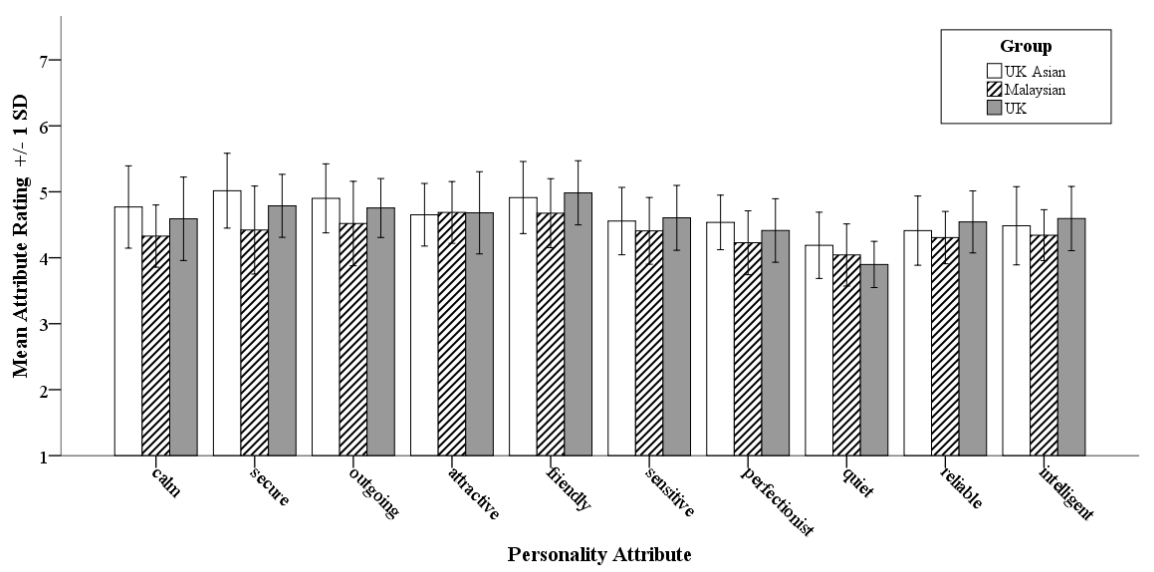

Fig. 1. Mean ratings ( $\pm 1 \mathrm{SD}$ ) of each personality attribute among the three cultural groups.

\subsection{Relationship of profile owners' personalities with raters' own personality.}

In order to examine the impact of observers' own personalities when judging others, Pearson correlation coefficients were calculated separately for the UK group, Malaysian group and UK Asian group with averaged personality assessments of the profile owners' attributes (Table 3).

Table 3. Pearson correlations between raters' own personalities and averaged ratings of profile owners $(* \mathrm{p}<.05 * * \mathrm{p}<.01)$.

\begin{tabular}{lccc}
\hline Attribute & $\begin{array}{c}\text { UK } \\
\text { Western }\end{array}$ & Malaysian & $\begin{array}{c}\text { UK } \\
\text { Asian }\end{array}$ \\
\hline Nervous-Calm & .13 & $.45^{*}$ & .04 \\
Insecure-Confident & .04 & .15 & .06 \\
Shy-Outgoing & -.09 & $.44^{*}$ & .01 \\
Unattractive-Attractive & -.18 & -.02 & .02 \\
Unfriendly-Friendly & $.39^{*}$ & $.38^{*}$ & .30 \\
Insensitive-Sensitive & .27 & .26 & .34 \\
Careless-Perfectionist & $.43^{* *}$ & -.10 & -.02 \\
Quiet-Loud & -.11 & .03 & .20 \\
Unreliable-Reliable & $.37^{*}$ & .21 & .18 \\
Unintelligent-Intelligent & .25 & .12 & .07 \\
\hline
\end{tabular}


In the UK group, significant correlations were found for judgments of friendliness $(r=.39)$, perfectionist $(r=.43)$ and reliability $(r=.37)$. In other words, UK raters who rated themselves higher on these traits, tended to rate others who showed those traits higher as well.

In the Malaysian group, a different pattern of relationships emerged. Significant correlations were found between observers' and owners' ratings of calmness $(\mathrm{r}=.45)$, outgoingness $(\mathrm{r}=.44)$ and friendliness $(\mathrm{r}=.38)$. This suggests that Malaysian students who had never been to the UK who considered themselves to be more calm, outgoing and friendly were more likely to assume these traits in others. In contrast, no relationships were found between UK-based Asian students' personality and the averaged ratings of others' personality.

Collectively, these findings suggest that there may be a limited impact of one's own personality on the judgment of others, but that this effect is different across cultural backgrounds. Where significant correlations existed, it suggests that when one rates themselves higher on a trait, they will subsequently rate others higher on that particular trait as well. In other words, when people are unsure of making judgments of others, they may tend to judge others based on their own personality traits.

\section{Discussion}

The current study demonstrates that when making judgments of others' personality at zero-acquaintance, first impressions are not necessarily accurate even when judged from the same cultural background. Among the three groups of observers, individuals were only able to judge approximately one out of ten personality traits accurately regardless of their culture origin; sensitivity was most accurately judged by the UK group while friendliness was most accurately judged by the Malaysian and UK Asian groups. The hypothesis that people from similar cultural backgrounds would form more accurate first impressions was therefore not supported. This finding appears inconsistent with previous studies [12] that have suggested accurate impressions can be formed after brief initial exposure to strangers via social media. However, their studies included more detailed tasks such as browsing full profiles that might enable one to gain more information on which to form impressions, rather than just being exposed briefly to profile pictures. This might suggest that social media profile pictures alone do not necessarily provide sufficient information for making accurate judgments of non-visible personality attributes.

Past studies [9] have suggested that accuracy of judgments can be achieved after approximately 60 seconds of exposure for face-to-face situations, which is longer than the exposure duration used in the current study. It is important to note that the selected exposure was implemented to better understand if accuracy could be achieved more promptly via social media, given the short dwell time typically spent by users of SNS when first viewing online information about others. It should be taken into account that increasing exposure time could have led to better accuracy overall.

Despite this, whilst there was some variation between cultural groups, a consistent finding in the present study was that there tended to be good agreement between raters (consensus) regarding the opinions they formed of others. For example, good consensus between raters was found for attractiveness in the UK group as well as the 
Malaysian group. This could suggest that standards of attractiveness to a certain extent are consistent between these two cultures. However, low agreement for attractiveness was found in the UK Asian group who it could be argued may have had greater exposure to Western culture. This may suggest that although one is exposed to a culture, it cannot be assumed that traits will be judged consistently to natives of that culture, or that interaction with individuals in a foreign culture may affect people's perception of traits such as attractiveness differently. Alternatively, it could be that the UK Asian group used in the present study did not represent one homogeneous group but contained individuals drawn from a variety of countries, which may hold different values of attractiveness. Although observer consensus was relatively strong overall within each cultural group, self-other agreement ratings were low, reflecting that consensus does not appear to always be an index of accuracy when forming impressions [16].

Previous research has suggested that people from eastern cultures may perceive smiles differently, due to different display rules [7]. Despite this, findings from the current study suggested that both Malaysian and Asian groups were more accurate in judging friendliness and tended to rate Western profile owners as more friendly, when assessed from profile pictures in which the majority of profile owners $(67 \%)$ were smiling. Undoubtedly, the Malaysian and UK Asian groups may show different cultural expectations compared to Japanese. This perhaps implies that it is possible to judge friendliness in other cultures even where different social display rules may exist. Alternatively, since 'true' levels of friendliness were based only on the profile owners own view as to how friendly they were, it is possible that self-other agreement was artificially inflated by profile owners' tendency to overestimate their own level of friendliness.

An unexpected finding was that people from different cultural backgrounds differed with respect to the absolute levels with which they rated personality attributes. Since all participants rated the same set of target stimuli, it can be assumed that there may be consistent cultural differences in how they see others on several personality attributes. In the present study, such stereotypical differences between cultures were found with respect to the attributes of confidence, calmness, outgoingness, quietness, friendliness and being a perfectionist. Our results also suggested that an individual's own personality exerts an impact when making judgments of others as shown by the UK and Malaysian group. This may be partially explained by Human and Biesanz [17] who demonstrated that although well-adjusted individuals may not able to judge unique characteristics accurately; they tend to judge others based on assumed attributes of others or similarity to one's self. It is therefore possible that both normative accuracy (perceiving others as similar to the average person) and assumed similarity (perceiving others as similar to the self) may have limited the overall level of agreement between profile owners and raters in the present study.

That accuracy ratings did not differ between UK based Asians and those overseas is noteworthy. A possible explanation for this could be that UK Asian students currently residing in the UK are more likely to remain in their own cultural groups rather than integrating with the locals. Studies have shown that Chinese students studying abroad find it difficult to integrate with British students due to the differences in lifestyles and values [18]. It is therefore logical to assume when students do not integrate, they may be no more accurate at judging individuals in their new culture than individuals who have never been exposed to that culture. 
In conclusion, the present study suggests that whilst consensus may be relatively high when it comes to making personality judgements about others about whom we have little information, overall accuracy when compared to the person's evaluation of their own personality tends to be low, regardless of cultural background. Taken together our findings suggest that people from all cultural backgrounds may rely more on their own personality and beliefs about the 'typical' personality of others when forming first impressions and that greater cultural similarity does not necessarily lead to more accurate perceptions of others based on brief initial exposures.

Acknowledgments. With kind thanks to Help University, Wilayah Persekutuan, Kuala Lumpur, Malaysia for their assistance with the conduct of this project.

\section{$5 \quad$ References}

1. Ambady, N., \& Rosenthal, R. (1992). Thin Slices of Expressive Behavior as Predictors of Interpersonal Consequences: A Meta-Analysis. Psychology Bulletin, 111(2), 256-274.

2. Johnston, K., Chen, M., \& Hauman, M. (2013). Use, Perception and Attitude of University Students towards Facebook and Twitter. Electronic Journal of Information Systems Evaluation, 16(3), 200-210.

3. Zhao, C., \& Jiang, G. (2011). Cultural differences on visual self-presentation through social networking site profile images. CHI '11 Proceedings of the SIGCHI Conference on Human Factors in Computing Systems, 1129-1132. doi:10.1145/1978942.1979110

4. Triandis, H. C. (1995). Individualism \& collectivism. Boulder, CO: Westview Press.

5. Chau, P. Y. K., Cole, M., Massey, A. P., Montoya-Weiss, M., \& O'Keefe, R. M. (2002). Cultural Differences in the Online Behavior of Consumers. Communications of the ACM, 45(10), 138-143.

6. Kim, Y., Sohn, D., \& Choi, S. M. (2011). Cultural difference in motivations for using social network sites: A comparative study of American and Korean college students. Computers in Human Behavior, 27(1), 365-372. doi:10.1016/j.chb.2010.08.015

7. Matsumoto, D., \& Kudoh, Tsutomu. (1993). American-Japanese Cultural Differences in Attributions of Personality Based On Smiles. Journal of Nonverbal Behavior, 17(4), 231-243.

8. Uleman, J. S. (1999). Spontaneous versus intentional inferences in Impression formation. In S. Chaiken, \& Y.Trope (Eds.), Dual-Processes Theories in Social Psychology (pp. 141-160). New York: Guildford Press.

9. Carney, D. R., Colvin, C. R., \& Hall, J. A. (2007). A thin slice perspective on the accuracy of first impressions. Journal of Research in Personality, 41(5), 1054-1072.

10. Naumann, L. P., Vazire, S., Rentfrow, P. J., \& Gosling, S. D. (2009). Personality Judgments Based on Physical Appearance. Personality and Social Psychology Bulletin, 35(12), 16611671.

11. Steecher, K., \& Counts, S. (2008). Thin slices of online profile attributes. Proceedings of ICWSM, 8.

12. Latisha Asmaak Shafie, Surina Nayan, \& Nazira Osman. (2012). Constructing Identity through Facebook Profiles: Online Identity and Visual Impression Management of University Students in Malaysia. Procedia - Social and Behavioral Sciences, 65(3), 134-140. doi:10.1016/j.sbspro.2012.11.102

13. Hum, N. J., Chamberlin, P. E., Hambright, B. L., Portwood, A. C., Schat, A. C., \& Bevan, J. L. (2011). A picture is worth a thousand words: A content Analysis of Facebook profile photographs. Computers in Human Behavior, 27(5), 1828-1833. 
14. Turner, M, Hunt, N. (2014). What does your profile picture say about you? The accuracy of thin-slice personality judgments from social networking sites made at zero-acquaintance. Social computing and social media: 6th International Conference, SCSM 2014, Held as Part of HCI International 2014, Heraklion, Crete, Greece, June 22-27, 2014. In Meiselwitz, G. (ed.). Springer, p. 506-516 (Lecture notes in computer science; vol. 8531)

15. Bond, M. H. (1978). Dimensions used in perceiving peers: Cross-cultural comparisons of Hong Kong, Japanese, American and Filipino university students. International Journal of Psychology, 14(1), 47-56.

16. Rule, N., \& Ambady, N. (2010). First Impressions of the Face: Predicting Success. Social and Personality Psychology Compass, 4(8), 506-516.

17. Human, L. J., \& Biesanz, J. C. (2011). Through the Looking Glass Clearly: Accuracy and Assumed Similarity in Well-Adjusted Individuals' First Impressions. Journal of Personality and Social Psychology, 100(2), 349-364.

18. Spencer-Oatey, H., \& Xiong, Z. (2006). Chinese Students' Psychological and Sociocultural Adjustments to Britain: An Empirical Study. Language, Culture and Curriculum, 19(1), 3753. 